\title{
COVID-19 and Stroke Risk: A Double Whammy
}

\author{
Alvee Saluja ${ }^{1} \quad$ Rajinder K. Dhamija ${ }^{1}$ \\ 1Department of Neurology, Lady Hardinge Medical College, \\ New Delhi, India
}

Ann Natl Acad Med Sci (India):2020;2:58-61

\begin{abstract}
Address for correspondence Rajinder K. Dhamija, MD, DNB (Neuro), FRACP, FRCP, Department of Neurology, Lady Hardinge Medical College, New Delhi 110001, India (e-mail: dhamijark@gov.in).
\end{abstract}

\begin{abstract}
The emphasis so far during the COVID-19 pandemic has been on the respiratory manifestations with little attention being given to neurological manifestations. Literature has shown multiple cases of stroke being associated with COVID-19. Thus, there is great interest in the role of the virus in stroke pathogenesis. Regarding hyperacute and acute stroke treatment, the routine guidelines for thrombolysis and thrombectomy are to be followed with emphasis on high suspicion of COVID-19 in stroke cases with respiratory symptoms or with contact/travel history. Secondary risk factor treatment for hypertension, diabetes, dyslipidemia is a must. We recommend continuing angio-

Keywords

- COVID-19

- stroke

- SARS-CoV-2

- risk factors

- pandemic tensin converting enzyme 2 inhibitors/angiotensin II receptor blockers (ARBs) in those who are taking these medications as per evidence available. Mandatory lockdown has led to delay in presentation to the hospital with a decrease in thrombolysis due to ineligibility and a corresponding increase in primary thrombectomies being performed. Telemedicine could be an important tool to triage cases worthy of tertiary referral from other strokes and must be encouraged.
\end{abstract}

\section{Introduction}

The novel coronavirus disease 2019 (COVID-19) caused by SARS-Cov-2 virus, was declared a pandemic by the WHO (World Health Organization) on March 11, 2020. This was just two and a half months after the first case was reported on December 2019 in Wuhan, China. This just demonstrates the rapid human transmissibility of this virus which has spread across continents in such a brief period of time. At the time of writing this article, COVID-19 has already infected 2,726,724 cases worldwide and led to more than 191,000 deaths worldwide. The number of cases in India are still on the rise. Hence, it becomes all the more important for us to know as much as possible regarding the varied manifestations of this virus. The emphasis so far has only been on the predominant respiratory manifestations of this illness and scant literature or only anecdotal case reports are available regarding the neurological manifestations of COVID-19. These neurological manifestations will further add to the morbidity and mortality caused by this disease. In a recent retrospective case series of 214 Chinese patients, $36.4 \%$ of the cases had neurological manifestations. Out of the 214 patients, six (2.8\%) had evidence of stroke (ischemic or hemorrhagic). Strokes were common in patients with severe COVID-19 disease manifestations with five out of the six strokes (83.3\%) occurring in severely affected individuals. ${ }^{1}$

\section{Stroke and COVID-19: The Evidence So Far}

Both Stroke and COVID-19 impart greater morbidity and mortality among the elderly and those with co-morbidities like hypertension and diabetes mellitus. Hence, these groups are at a heightened risk for this double hit. ${ }^{2}$ Therefore for neurologists, it becomes important to be aware of the relationship between COVID-19 and stroke to suspect, quickly identify, quarantine, and control the spread of this disease to other non-COVID patients. In the study by Mao et al, cases of COVID-19 with central nervous system (CNS) manifestations also demonstrated a significantly lower lymphocyte and platelet count with an elevated blood urea nitrogen. ${ }^{1}$ Also, severely affected COVID-19 cases had significantly higher C-reactive protein (CRP) and D-dimer levels which hints at a pro-inflammatory cascade probably due to increased cytokine release in severe cases. ${ }^{1}$ 
The occurrence of diffusion abnormalities found in the MRI brain of two patients with severe COVID-19 who did not have any focal neurological deficits further raises the question of a link between COVID-19 infection and occurrence of stroke. ${ }^{3}$ Subsequently, a case report from Iran described lobar intracranial hemorrhage in a 79-year-old male without co-morbidities who had developed loss of consciousness after 3 days of fever and cough. Interestingly, the patient had a normal coagulation profile on admission and tested positive for COVID-19. ${ }^{4}$ However, it is important to note that some cases of stroke with concomitant COVID-19 reported so far have not presented with typical flu like symptoms. These cases presented as focal neurological deficits and lung pathology were subsequently detected on emergency chest computed tomography (CT) scans. These cases developed cough, fever, and flu like symptoms later in the course, a few days after the onset of deficit. ${ }^{1}$ This presentation emphasizes the need for a higher suspicion among cases of acute ischemic stroke coming to the emergency.

In terms of mortality, case fatality rate has been estimated to be as high as $49 \%$ among severely affected and critically ill COVID-19 cases. A higher case fatality rate has also been reported independently with the presence of advanced age, hypertension, diabetes mellitus, ischemic heart disease, and prior ischemic stroke. ${ }^{5}$ Since, most of these co-morbidities are proven stroke risk factors, the higher occurrence of stroke and stroke-related deaths among COVID-19 cases is to be expected secondary to traditional stroke risk factors.

\section{Plausible Pathogenic Mechanisms for Increased Stroke Risk and COVID-19}

As such patients with stroke who traditionally are elderly and have comorbidities are already at an increased risk of getting infected with COVID-19. This forms the major chunk of co-occurring COVID-19 and stroke cases. However, atypical cases have prompted the study on the possible role of this viral infection in stroke pathogenesis. Studies on the various pathogenic mechanisms of COVID-19 have demonstrated role of a pro-inflammatory cascade with higher levels of CRP, interleukin 6, D-dimer, and multiple other cytokines in these patients. ${ }^{6}$ Prior studies have found an association of acute ischemic stroke with pro-inflammatory markers as well. ${ }^{7.8}$ The higher levels of these pro-inflammatory markers among COVID-19 patients have been associated with more severe manifestations, poorer outcomes, and a greater occurrence of neurological manifestations. Thus, a plausible unifying hypothesis could be a procoagulant state induced by the pro-inflammatory milieu, leading to a greater chance of vascular thrombosis among individuals who are already harboring traditional stroke risk factors. The occurrence of venous thromboembolism in up to $31 \%$ cases in a case series of 184 intensive care unit (ICU) patients with severe COVID-19 pneumonia also supports a hypercoagulable state due to an excessive inflammatory response among COVID-19. ${ }^{9}$ The low platelet count and elevated D-dimer consequent to immune dysregulation and the cytokine storm predispose these patients to develop intracranial bleeding as well. It is also known that a pro-inflammatory state due to immune activation can result in endothelial dysfunction which itself leads to an increased risk of vascular thrombosis. ${ }^{10}$ Another possible mechanism could be the role of angiotensin converting enzyme 2 (ACE2) receptor. ACE2 has now been recognized as the receptor through which virus binds to the host cells. ${ }^{11} \mathrm{ACE}$ and its receptors are present in the lungs, gastrointestinal tracts, nervous system, skeletal muscles, and in the cerebral endothelial cells. Interestingly, these are some of the major target organs of this virus. ${ }^{12}$ ACE2 converts angiotensin II into a heptapeptide (angiotensin [1-7]) having vasodilatory, antioxidant, and anti-inflammatory actions. ${ }^{13,14}$ There have been studies regarding downregulation of ACE2 post binding by COVID-19 to host cell membranes. Therefore, ACE2 downregulation might lead to vasoconstriction, pro-inflammatory effects leading to increased risk of hypertension and strokes. The finding of hypertension as the major co-morbidity among severe COVID-19 cases and among those with neurological manifestations could very well be explained by the ACE2 receptor hypothesis. Another possible mechanism could be due to increased cardiovascular diseases incidence (like acute myocardial infarction, valvular vegetations, and arrhythmias) precipitated by viral illness..$^{15}$ This would lead to a greater risk of cardioembolic strokes. Recently, the first case of meningitis with detection of viral ribonucleic acid (RNA) in cerebrospinal fluid in a COVID-19 patient was reported, hinting at a direct neurotropic role of this virus. ${ }^{16}$ Hence, locally mediated CNS inflammation and CNS vasculitis secondary to the virus could be possible mechanisms especially in those without typical flu-like symptoms. The mode of spread of the virus to the CNS may be through hematogenous route in cases with severe COVID-19 infection. However, a plausible mechanism could be through trans ethmoid spread via olfactory rootlets as case series have reported anosmia as an early manifestation of COVID-19 disease. ${ }^{17,18}$ This might explain some cases presenting as neurological manifestation prior to development of respiratory complaints. However, more studies and the association of olfactory symptoms among those presenting as strokes due to COVID-19 will have to be studied before any conclusion can be made. Thus, severe COVID-19 infection could tilt the balance toward developing a stroke in those who already have traditional risk factors.

\section{Hyperacute and Acute Management of Stroke Patients with COVID-19}

The occurrence of acute ischemic stroke at the time of this pandemic poses a great challenge regarding emergency treatment (particularly administering thrombolysis and performing thrombectomy) of acute stroke patients. From the time the patient arrives in the emergency, it would be pertinent to do a quick screen for respiratory symptoms and travel history. Quick transportation for CT scan through fast tracked low traffic corridors with prior communication to the radiology colleagues is of utmost importance as well. The presence of concomitant COVID-19 infection in stroke patients is not a contraindication for thrombolysis per se unless there is evidence of coagulopathy or disseminated intravascular 
coagulopathy (DIC) due to severe infection. The risk of post thrombolysis bleed will depend on the coagulation profile of the patient as it may be deranged in severe COVID-19 cases due to excessive cytokine release. Hence, acute treatment for those who deserve thrombolysis is to be performed but with a special care toward ruling out sepsis, DIC, thrombocytopenia, and donning adequate personal protective equipment (PPE) while sampling and cannulation.

With regards to thrombectomy, there must be adequate information provided beforehand to the neurointervention team if a stroke patient is demonstrating any respiratory symptoms, so that adequate PPE can be donned. There is mechanistically no difference in the technique of thrombectomy to be performed apart from high-risk prognosis for those with COVID-19 and respiratory distress. The site of post thrombolysis or thrombectomy care would be of utmost importance to prevent the spread of infection to other neurological patients. Hence, a dedicated COVID-19 stroke ward/ ICU would be ideal for isolating these patients while imparting optimal care.

The routine control of hypertension (those taking ACE inhibitors/ARBs should continue taking these medications), ${ }^{19}$ diabetes, fever, and care of post stroke swallowing care are necessary in all stroke patients with or without COVID-19 if we are to achieve good stroke outcomes. Regarding care of severe COVID-19 stroke patients who are in ICU, or who are immobilized should receive deep vein thrombosis prophylaxis routinely because of increased risk of venous thromboembolism. ${ }^{9}$

\section{Implications of the Lockdown Due to COVID-19 for Stroke Patients}

The sheer pace with which this virus has spread has forced governments to take drastic steps. Thus, patients are reluctant or unable to avail medical care due to the lockdown, restricted social mobility, suspended OPDs, and suspension of public/private transport systems. This is particularly important for acute stroke patients where even a slightest delay can be catastrophic having an impact on morbidity and mortality. Recently few examples have come up where patients have ignored stroke symptoms only to develop a severe deficit the next morning. ${ }^{20} \mathrm{~A}$ recent communication with Italy also reflected on the dwindling number of acute stroke cases to the emergency room. ${ }^{21}$ Reports from a tertiary care center in Italy also recorded a 26to 30\% decrease in combined thrombolysis and thrombectomy rates. However, they also had a $41 \%$ increase in primary thrombectomy cases which was due to transportation delay and patients being ineligible for thrombolysis. ${ }^{22}$ Therefore, alternate strategies must be sought for tackling these specific issues. Providing services through telemedicine consultations would lead to prompt recognition of symptoms and triaging cases requiring tertiary care. The efficacy of this process has been proven in various neurological diseases around the globe. ${ }^{23-25}$ Spreading awareness among the community regarding prevailing emergency services and tertiary stroke centers capable of comprehensive acute stroke treatment would be another step.

\section{Future Direction for Stroke Care in COVID-19 Patients in the Indian Scenario}

The finding of neurological manifestations in the absence of respiratory findings portends a great challenge for neurologists and emergency physicians. It also puts other admitted neurology and ICU patients at a greater risk of developing a highly infectious disease. Care of such stroke patients with concomitant COVID-19 infection would be especially challenging due to time constraints in treatment, multiple health care professionals involved (nurses, emergency physicians, neurologists, radiologists), transportation to various hospital areas, and limited availability of PPE.

Therefore, to tackle this situation, neurologists need to be aware and should have a high index of suspicion of COVID-19 among stroke cases especially at the peak of this pandemic. Second, quick transportation of stroke patients and well-defined stroke pathways would be the need of the hour. Such COVID-specific stroke pathways have already been designed in certain countries. ${ }^{22}$ Radiological investigations like CT/CT angiography for COVID-19 patients could be done via mobile units or in designated hospital areas specific to COVID-19 patients. Routine PPE use by neurologists taking care of acute stroke case with respiratory symptoms will help as well. Considering testing of stroke patients presenting with respiratory complaints or developing symptoms late in hospital course will help in greater case detection, isolation, and prevention of disease transmission among vulnerable hospitalized patients. All these preventive measures might help us in delivering better stroke care to patients during these times. Post COVID-19 follow-up and further insights into pathophysiology and long-term outcome of stroke patients will be of paramount importance. Till such time, neurologists and physicians caring for stroke patients, need to be sensitized about the stroke occurring in the context of COVID-19 and its implications for acute stroke treatment, secondary stroke prevention, and stroke rehabilitation.

\section{Conflict of Interest}

None declared.

\section{References}

1 Mao L, Jin H, Wang M, et al. Neurologic manifestations of hospitalized patients with coronavirus disease 2019 in Wuhan, China. JAMA Neurol 2020;77(6):1-9

$2 \mathrm{Wu} \mathrm{Z,} \mathrm{McGoogan} \mathrm{JM.} \mathrm{Characteristics} \mathrm{of} \mathrm{and} \mathrm{important} \mathrm{les-}$ sons from the Coronavirus Disease 2019 (COVID-19) Outbreak in China: summary of a report of 72314 cases from the Chinese Center for Disease Control and Prevention. JAMA 2020;323:1239-1242

3 Helms J, Kremer S, Merdji H, et al. Neurologic features in severe SARS-CoV-2 infection. N Engl J Med 2020. Doi: 10.1056/ NEJMc2008597

4 Sharifi-Razavi A, Karimi N, Rouhani N. COVID-19 and intracerebral haemorrhage: causative or coincidental? New Microbes New Infect 2020;35:100669

5 Xie J, Tong Z, Guan X, Du B, Qiu H. Clinical characteristics of patients who died of coronavirus disease 2019 in China. JAMA Netw Open 2020;3(4):e205619

6 Li X, Geng M, Peng Y, Meng L, Lu S. Molecular immune pathogenesis and diagnosis of COVID-19. J Pharm Anal 2020. Doi: 10.1016/j.jpha.2020.03.001 
7 Tuttolomondo A, Di Raimondo D, di Sciacca R, Pinto A, Licata G. Inflammatory cytokines in acute ischemic stroke. Curr Pharm Des 2008;14(33):3574-3589

8 Doll DN, Barr TL, Simpkins JW. Cytokines: their role in stroke and potential use as biomarkers and therapeutic targets. Aging Dis 2014;5(5):294-306

9 KlokFA, Kruip MJHA, van der Meer NJM, et al. Incidence of thrombotic complications in critically ill ICU patients with COVID-19. Thromb Res 2020. Doi: 10.1016/j.thromres.2020.04.013

10 Cooke JP. The endothelium: a new target for therapy. Vasc Med 2000;5(1):49-53

11 Zhao Y, Zhao Z, Wang Y, Zhou Y, Ma Y, Zuo W. Single-cell RNA expression profiling of ACE2, the putative receptor of Wuhan 2019-nCov. bioRxiv 2020. Doi: https://doi. org/10.1101/2020.01.26.919985

12 Hamming I, Timens W, Bulthuis ML. Lely AT, Navis G, van Goor $\mathrm{H}$. Tissue distribution of ACE2 protein, the functional receptor for SARS coronavirus. A first step in understanding SARS pathogenesis. J Pathol 2004;203(2):631-637

13 Santos RA. Angiotensin-(1-7)Hypertension 2014;63(6):1138-1147

14 El-Hashim AZ, Renno WM, Raghupathy R, Abduo HT, Akhtar S, Benter IF. Angiotensin-(1-7) inhibits allergic inflammation, via the MAS1 receptor, through suppression of ERK1/2- and NF-KBdependent pathways. Br J Pharmacol 2012;166(6):1964-1976

15 Madjid M, Safavi-Naeini P, Solomon SD, Vardeny O. Potential effects of coronaviruses on the cardiovascular system: a review. JAMA Cardiol 2020. Doi: 10.1001/jamacardio.2020.1286

16 Moriguchi T, Harii N, Goto J, et al. A first case of meningitis/ encephalitis associated with SARS-Coronavirus-2. Int J Infect Dis 2020;94:55-58
17 Giacomelli A, Pezzati L, Conti F, et al. Self reported olfactory and taste disorders in SARS-CoV-2 patients: a cross-sectional study. Clin Infect Dis 2020. Doi: 10.1093/cid/ciaa330

18 HopkinsC, KumarN2020. Loss of sense of smell as marker of COVID-19 infection. Available at: https://www.entuk. org/sites/default/files/files/Loss\%20of\%20sense \%20of\%20 smell\%20as\%20marker\%20of\%20COVID.pdf

19 Li J, Wang X, Chen J, Zhang H, Deng A. Association of renin-angiotensin system inhibitors with severity or risk of death in patients with hypertension hospitalized for coronavirus disease 2019 (COVID-19) infection in Wuhan, China. JAMA Cardiol 2020. Doi: 10.1001/jamacardio.2020.1624

20 Zhao J, Rudd A, Liu R. Challenges and potential solutions of stroke care during the coronavirus disease 2019 (COVID-19) outbreak. Stroke 2020;51(5):1356-1357

21 Morelli N, Rota E, Terracciano C, et al. The baffling case of ischemic stroke disappearance from the casualty department in the COVID-19 era. Eur Neurol 2020. Doi: 10.1159/000507666

22 Baracchini C, Pieroni A, Viaro F, et al. Acute stroke management pathway during coronavirus-19 pandemic. Neurol Sci 2020;9:1-3

23 Cohen BH, Busis NA, Ciccarelli L. Coding in the world of COVID19: non-face-to face evaluation and management. Continuum: Lifelong Learn Neurol-Neurol Syst Dis 2020;26(3):1-25

24 Guzik AK, Switzer JA. Teleneurology is neurology. Neurology 2020;94(1):16-17

25 Wilson AM, Jamal NI, Cheng EM, et al. Teleneurology clinics for polyneuropathy: a pilot study. J Neurol 2020;267(2):479-490 